\title{
Application of Optical Spectrophotometry for Analysis of Radiation Spectrum Emitted by Electric Arc in the Air
}

\author{
Michał Koziol, Daria Wotzka, Tomasz Boczar, and Paweł Frącz \\ Faculty of Electrical Engineering, Automatic Control and Computer Science, Opole University of Technology, \\ Prószkowska 76, 45-758 Opole, Poland
}

Correspondence should be addressed to Daria Wotzka; daria@wotzka.eu

Received 26 October 2016; Accepted 6 December 2016

Academic Editor: Javier Garcia-Guinea

Copyright (C) 2016 Michał Kozioł et al. This is an open access article distributed under the Creative Commons Attribution License, which permits unrestricted use, distribution, and reproduction in any medium, provided the original work is properly cited.

This paper presents the results of measurement and analysis of optical radiation emitted by a free burning electric arc. The aim was to determine the application possibilities of optical spectrophotometry for detection of electric arcs. The research works considered electric arc generated with a constant voltage supply between two copper electrodes in the air, carried out under laboratory conditions. A high resolution optical spectrophotometer was used for registration of optical radiation. The analyses involved determination of two dimensionless descriptors obtained for the gathered spectra. Moreover, for each of the registered intensity distributions, the energy values were calculated for three frequency ranges. Based on the measured signals, the possibility of application of spectrophotometry for the optical radiation analysis was confirmed. The analysis indicated that the most energy of optical radiation is detected for the range of $200-780 \mathrm{~nm}$, while above $780 \mathrm{~nm}$ almost no optical energy is emitted. Spectrophotometric studies performed in the UV-NIR range are of interest since one can obtain information about the structural defects (at lower wavebands) or impurities and/or point defects (at low energies bands). It was also stated that the obtained descriptors may be applied for diagnosis and identification of electric arc purposes.

\section{Introduction}

In general, an electric arc is a phenomenon occurring during the discharge of electricity. Apart from its useful technical applications in various branches of science and industry, it sometimes has a negative impact on systems or devices, as is the case of, for example, electric power equipment. Mostly, it occurs by contact elements of switching devices that operate under high voltage. Such devices are usually protected against the occurrence of electrical arcs. They are featured with various types of systems enabling limiting and extinguishing of an arc. However, an electric arc may occur at locations where damage or failure of insulating elements in electric power equipment exists. In such cases, appearance of an arc is often an undesirable effect. Beside the technical sources of electric arcs, such as structural or material defects, there are also failures resulting from human error.

Hence, modern electric arc safety systems have been developed and implemented with the aim of detecting an electric arc occurrence and protecting the device by switching it off as quickly as possible. These systems, among others, are based on detectors of light emitted by the arc, which is one of the criteria necessary for the system to be activated. The device monitors light intensity in the protected area and when its limit is exceeded, the safety system is activated. The conducted research aiming at the improvement of practical applications of an electric arc is greatly focused on welding technology and contact systems of electric devices [1-3]. In [4] the authors present selected phenomena in the melt arc. They discuss theoretical thermal models of an electric arc and methods of estimating voltage drops on switchgear contacts. Furthermore, mathematical and physical models of contacts erosion were also presented. Other works consider numerical analysis and simulation of electric arc presented, for example, in [5-8].

Spectrophotometric studies performed in the UV-NIR range are of interest since one can obtain information about the structural defects (at lower wavebands) or impurities and/or point defects (at low energies bands) [9]. 


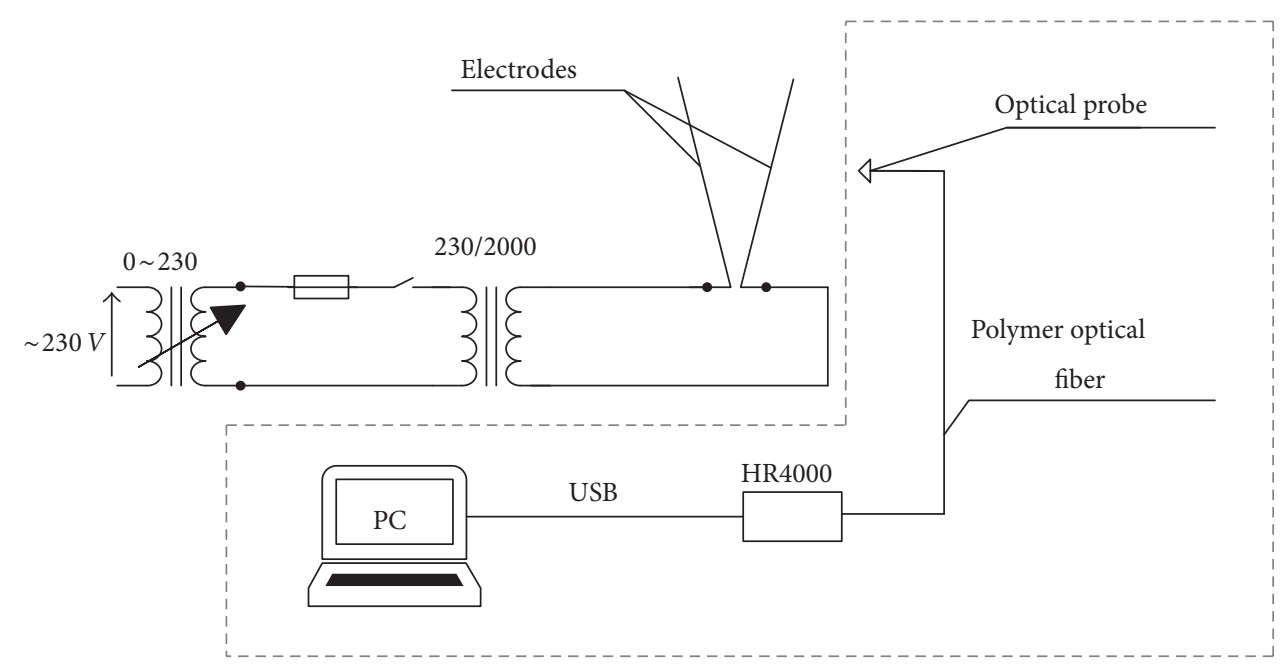

FIGURE 1: General diagram of the measurement system.

The aim of research works, the results of which are presented in this paper, was to determine the application possibilities of optical spectrophotometry for detection of an electric arc and to analyze the measured optical spectra within the range from 200 to $110 \mathrm{~nm}$ by supplying various voltage levels. For this purpose, a spectrophotometer of high optical resolution was used. Nowadays, the optical spectrophotometry method is frequently used for the electric arc analysis. For example, in $[10,11]$, this method was used for determining the temperature of an electric arc burning in a slot between contacts of electric devices. In [12, 13], optical spectrophotometry was chosen for analysis of optical spectra emitted by partial discharge in the air and oil insulation. In $[14,15]$, a spectrophotometer was applied for detection and analysis of surface discharges occurring on bushing and support insulator models under laboratory conditions. The spectroscopy method was also applied for studies of plasma [16-18].

The main contribution of this paper is in (1) presentation and discussion of the measured optical spectra that was emitted by a free burning electric arc generated by various voltage values, (2) indication of the dominant wavelength contained in the measured signals, (3) calculation of two dimensionless factors: peak and shape coefficients, which may be used for diagnosis purposes, and (4) calculation of the energy content in three spectral ranges of the electromagnetic radiation: ultraviolet (UV), visible light (VIS), and near infrared (NIR).

\section{Materials and Methods}

The research was conducted on a prototype measurement circuit composed of two copper electrodes arranged into $\mathrm{V}$ shape, which is commonly known as "Jacob's ladder." The circuit was supplied with various voltage values within the range of $1.5-1.9 \mathrm{kV}$ and was used for generation of a free burning electric arc, initiated manually. The measurements of the free burning electric arc were carried out in the atmospheric air.

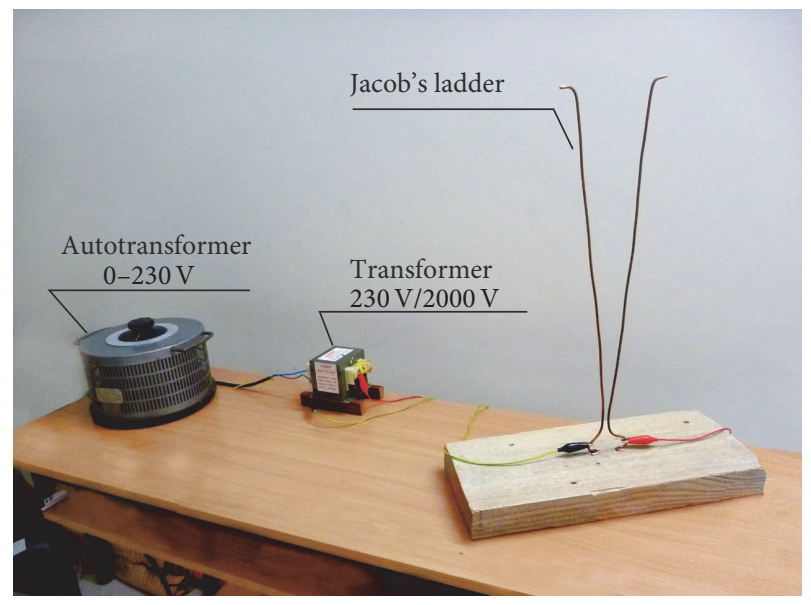

FIgure 2: Picture of the measuring system depicting the object under study.

A general diagram of the experimental circuit is depicted in Figure 1. A picture of the measuring system showing elements of the experimental setup is presented in Figure 2.

The radiation emitted by an electric arc was recorded using a high resolution optical spectrophotometer type HR4000 [19]. The device enables registration of optical signals in the range of UV-VIS-NIR. The spectrophotometer is equipped with a charge coupled device that allows for detection of 3648 components with dimension $8 \times 200 \mu \mathrm{m}$ each. An example snapshot is presented in Figure 3, depicting an electric arc and assembly method of the optical fibre. The acceptance angle of the optical sensor is also marked.

The measurement procedure of a spectrophotometer consists of counting the photons within a fixed time period $t$, provided that, at a single count, various number of photons is considered. The manufacturer of HR4000 provides the following information: (1) at $400 \mathrm{~nm}$, the single intensity count corresponds to 130 photons detected; (2) at $600 \mathrm{~nm}$, the 


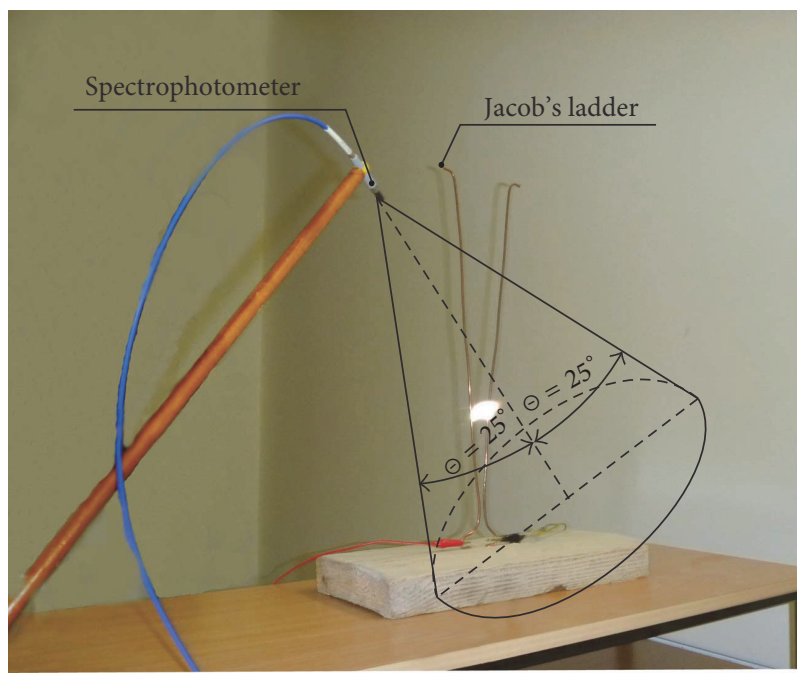

FIgURE 3: Picture of the object under study and optical fibre assembly. The acceptance angle of the optical sensor is $50 \mathrm{deg}$.

single intensity count corresponds to 60 photons detected. As measurement result, the device delivers a number of intensity counts for particular wavelengths and assigns it as intensity. The HR4000 device has an optical resolution at the level of $0.03 \mathrm{~nm}$ for the half-value width and operates in the spectral range from 200 to $1100 \mathrm{~nm}$ with the possibility of changing the spectral range and optical resolution depending on the selected grid and the input slot (aperture). Programming the time period $t$ in the range from $10 \mu \mathrm{s}$ to $65 \mathrm{~s}$ makes it possible to take measurements of short- and longduration events and phenomena. In the considered research works, all measurements were performed by constant time period $t$ equal to $1 \mathrm{~s}$. The device can be operated with the use of a dedicated computer software in real time. A universal connector SMA905 enables plugging various types of optical fibres, diffusers, or measuring probes. In this research, an optical signal was transmitted to the spectrophotometer using the polymer optical fibre of diameter $600 \mu \mathrm{m}$ and spectral range of $200-1100 \mathrm{~nm}$. This type of fibre is characterized by attenuation equal to $150 \mathrm{~dB} / \mathrm{km}$, which results in a decrease of the signal power, without affecting its shape. With regard to relatively short length of the applied fibre $(2 \mathrm{~m})$, the impact of light attenuation in the fibre core has not been analyzed.

\section{Spectral Analysis of the Obtained Measurement Results}

Several registrations were performed for each voltage level from 1500 to $1900 \mathrm{~V}$. The measured signals revealed a repeatable form of spectral distributions. The values of characteristic and dominant wavelength components, corresponding to local peaks of increased intensity, were characterized by a constant repeatability. Thus, arithmetic mean was calculated for each measurement trial. Figure 4 presents the averaged intensities, registered in the UV-VIS-NIR range, for considered voltage levels.
TABLE 1: Dominant wavelength components [nm] occurring in the considered ranges.

\begin{tabular}{l}
\hline \multicolumn{3}{c}{$\begin{array}{c}\text { Value of the characteristic component }[\mathrm{nm}] \text { for the particular } \\
\text { range }\end{array}$} \\
\begin{tabular}{lcc} 
UV & VIS & NIR \\
\hline $\begin{array}{l}307.2,309.6,325.2,327.8, \\
373.9\end{array}$ & $\begin{array}{c}423,511,516,522,571,579, \\
590,767\end{array}$ & 794,810 \\
\hline
\end{tabular}
\end{tabular}

The averaged spectral distributions were subjected for further detailed analysis. In general, it should be observed that the highest intensity of radiation includes UV range of $200-380 \mathrm{~nm}$ and VIS range of $380-780 \mathrm{~nm}$, whereas the part of NIR range of $780-1100 \mathrm{~nm}$ is characterized by low radiation intensity. In Figures 5-7, the calculated mean spectrum distribution is depicted separately for the UV, VIS, and NIR range, respectively, for selected voltage value of arc generation. In Figures 5-7, the dominant wavelengths are marked.

Based on the analysis of spectral distributions, presented in Figures 5-7, the components of individual wavelengths of highest intensity (dominant) within the considered ranges were selected and presented in Table 1. The criterion for determining the dominant components for UV and VIS ranges included the intensity of their occurrence, which was characterized by the number of counts exceeding 2000 . With regard to a relatively low contribution of the radiation within NIR range, which was less than 600 in the whole range, the wavelengths with the greatest number of counts were adopted as characteristic components.

\section{Descriptors Characterizing the Considered Phenomenon}

In order to indicate and analyze significant differences in the gathered spectra, two dimensionless descriptors were calculated. They can provide unique comparative criteria while identifying the occurrence of the electric arc: (a) the peak factor $W(1)$, which expresses the ratio of the maximum value to the root-mean-square value of the spectrum, and (b) the shape factor $K(2)$ calculated as the ratio of root-meansquare value to mean value of the considered spectrum [19]. The factors $W$ and $K$ were calculated separately for the UVVIS-NIR ranges of emitted radiation and are presented in Table 2.

$$
W=\frac{I_{\mathrm{MAX}}}{I_{\mathrm{RMS}}}
$$

where $I_{\text {MAX }}$ is maximal value of optical spectrum intensity [-] and $I_{\mathrm{RMS}}$ is root-mean-square value [-], calculated according to dependence (3).

$$
K=\frac{I_{\mathrm{RMS}}}{I_{\mathrm{AVG}}}
$$




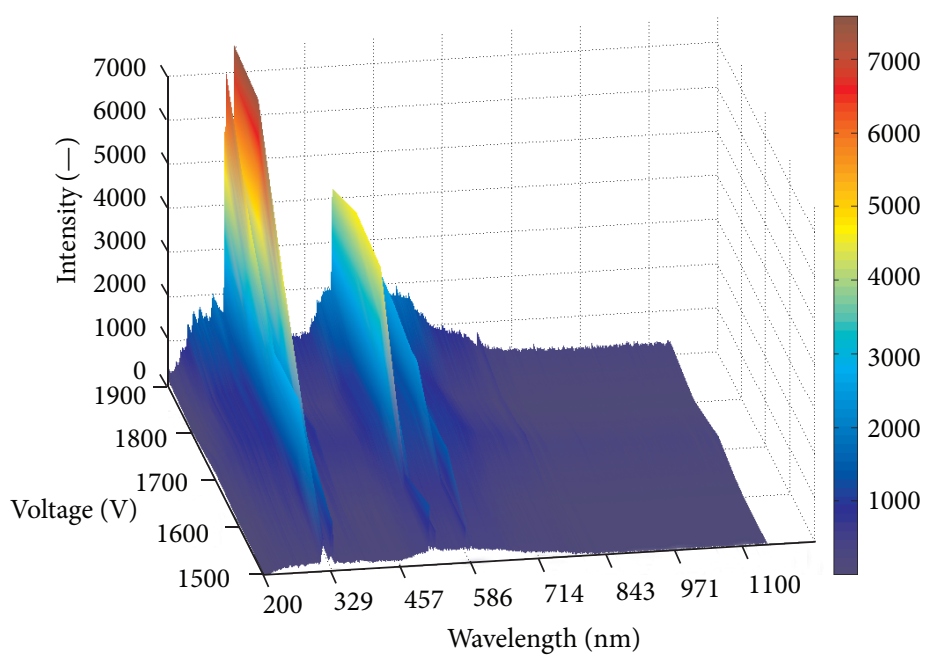

FigURE 4: Spectrogram of optical signals emitted by an electric arc by various voltage levels.

TABLE 2: Shape and peak factors calculated over registered spectra at the considered ranges.

\begin{tabular}{lcccccccc}
\hline \multirow{2}{*}{ Voltage [V] } & \multicolumn{2}{c}{ UV $(200 \mathrm{~nm}-380 \mathrm{~nm})$} & \multicolumn{2}{c}{ VIS $(380 \mathrm{~nm}-780 \mathrm{~nm})$} & \multicolumn{2}{c}{ NIR $(780 \mathrm{~nm}-1100 \mathrm{~nm})$} & \multicolumn{2}{c}{ Total range $(200 \mathrm{~nm}-1100 \mathrm{~nm})$} \\
& $W$ & $K$ & $W$ & $K$ & $W$ & $K$ & \multicolumn{1}{c}{$W$} & $\mathbf{6 . 5 9 5 7}$ \\
1500 & 4.5840 & 1.2486 & 5.1786 & 1.1108 & 3.1202 & 1.2264 & $\mathbf{1 . 3 5 5 7}$ \\
1600 & 4.5509 & 1.4524 & 3.5409 & 1.1368 & 2.9338 & 1.1579 & $\mathbf{7 . 2 0 6 6}$ & $\mathbf{1 . 5 1 0 2}$ \\
1700 & 4.5229 & 1.2104 & 5.0521 & 1.0907 & 1.9092 & 1.0280 & $\mathbf{6 . 0 9 8 3}$ & $\mathbf{1 . 2 5 5 2}$ \\
1800 & 5.6541 & 1.4728 & 5.7143 & 1.1833 & 3.3131 & 1.2412 & $\mathbf{9 . 3 9 8 3}$ & $\mathbf{1 . 6 2 8 4}$ \\
1900 & 3.9237 & 1.2896 & 2.8584 & 1.0867 & 2.0018 & 1.0252 & $\mathbf{5 . 8 7 4 2}$ & $\mathbf{1 . 3 3 9 9}$ \\
\hline
\end{tabular}

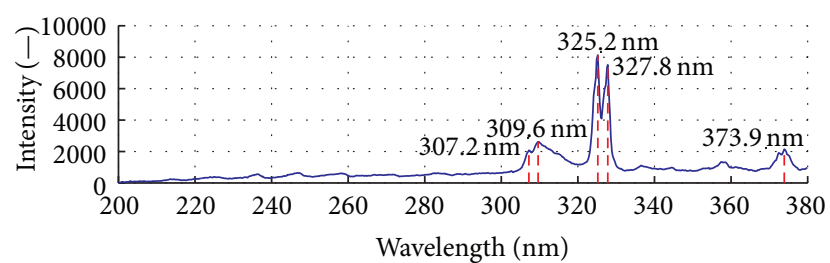

FIGURE 5: Spectral distribution depicting dominant components within the UV range.

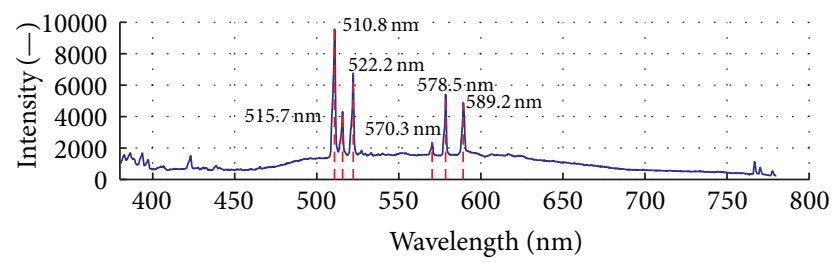

FIGURE 6: Spectral distribution depicting dominant components within the VIS range.

where $I_{\mathrm{AVG}}$ is mean value [-], calculated according to dependence (4).

$$
I_{\mathrm{RMS}}=\sqrt{\frac{\int_{f_{1}}^{f_{2}} I^{2}(f) d f}{\int_{f_{1}}^{f_{2}} d f}},
$$

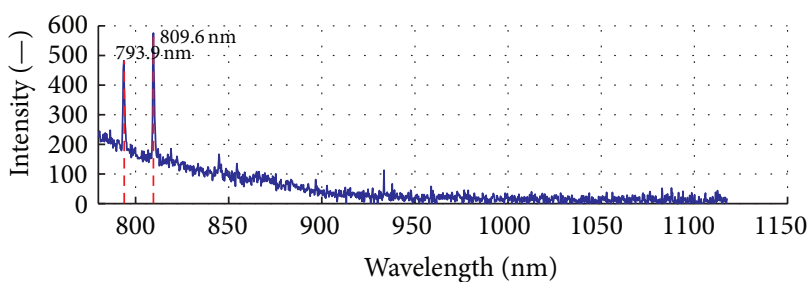

FIGURE 7: Spectral distribution depicting dominant components within the NIR range.

where $f_{1}$ is frequency at which the considered range begins $[\mathrm{Hz}]$ and $f_{2}$ is frequency at which the considered range ends $[\mathrm{Hz}]$.

$$
I_{\mathrm{AVG}}=\frac{\int_{f_{1}}^{f_{2}} I(f) d f}{\int_{f_{1}}^{f_{2}} d f} .
$$

For better analysis, Figure 8 presents the coefficients from Table 2 and also the mean is depicted as a solid line. It is to recognize that there is no significant dependency of voltage value on the $W$ and $K$ coefficients. The averaged values of both coefficients are the highest for the UV range. The peak factors for the UV and VIS ranges are very similar, while the lowest value was calculated for the NIR range. The shape factor for VIS and NIR is very similar and significantly lower as compared to the UV range. 
TABLE 3: The calculated electromagnetic radiation energy values.

\begin{tabular}{|c|c|c|c|c|c|c|c|}
\hline \multirow{2}{*}{ Voltage [V] } & \multicolumn{2}{|c|}{$E_{\mathrm{UV}}$} & \multicolumn{2}{|c|}{$E_{\mathrm{VIS}}$} & \multicolumn{2}{|c|}{$E_{\mathrm{NIR}}$} & \multirow{2}{*}{$\begin{array}{l}E_{C} \\
\text { pJ }\end{array}$} \\
\hline & $\mathrm{pJ}$ & $\% E_{C}$ & $\mathrm{pJ}$ & $\% E_{C}$ & pJ & $\% E_{C}$ & \\
\hline 1500 & 12.16 & 48.20 & 12.47 & 49.41 & 0.60 & 2.39 & 25.23 \\
\hline 1600 & 25.24 & 55.09 & 19.82 & 43.27 & 0.75 & 1.64 & 45.81 \\
\hline 1700 & 50.41 & 51.15 & 44.19 & 44.84 & 3.95 & 4.01 & 98.55 \\
\hline 1800 & 53.17 & 58.22 & 37.41 & 40.96 & 0.75 & 0.82 & 91.33 \\
\hline 1900 & 90.57 & 54.24 & 71.84 & 43.02 & 4.58 & 2.74 & 166.99 \\
\hline
\end{tabular}
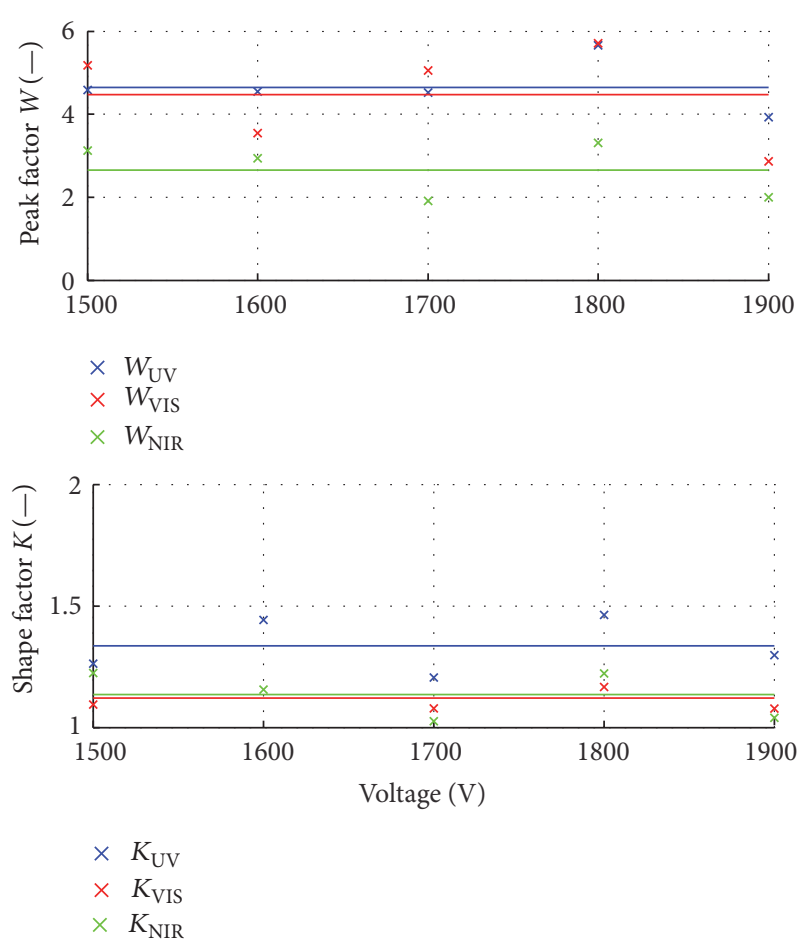

FIGURE 8: Shape and peak factors calculated over registered spectra at the considered ranges.

\section{Calculation of Energy Content in the Registered Signals}

The electromagnetic radiation frequency $f$ was determined using the following dependency:

$$
f=\frac{v}{\lambda}
$$

where $v$ is phase velocity, velocity of light $3 \cdot 10^{8}[\mathrm{~m} / \mathrm{s}], \lambda$ is wavelength $[\mathrm{m}]$, registered by spectrophotometer, and $f$ is electromagnetic radiation frequency $[\mathrm{Hz}]$.

The energy $E$ for single radiated photon is defined by [20]

$$
E=h f[\mathrm{~J}] \text {, }
$$

where $h$ is Planck constant $6.6 \cdot 10^{-34}$ [Js].

According to dependency (6), radiation energy is closely linked to the wavelength and its phase vibration frequency. Radiation energy increases along with the increase of wave frequency.
As mentioned in Section 2, the device given intensity is calculated as a number of photons registered at a particular wavelength. The authors of this study consider three ranges of electromagnetic radiation, but the producer defines the number of photons, considered as a single intensity count, only for two ranges: for the UV ( $n=130$ at $400 \mathrm{~nm})$ and upper VIS $(n=60$ at $600 \mathrm{~nm})$. Thus, for the purpose of further calculations, the following has been assumed: (1) the number of photons detected in the UV range equals $n_{\mathrm{UV}}=$ 130 , (2) the number of photons detected in the VIS range equals $n_{\mathrm{VIS}}=95$, and (3) the number of photons detected in the NIR range equals $n_{\mathrm{NIR}}=60$. The value $n_{\mathrm{VIS}}=95$ is an average for 130 and 60 .

Taking the above into consideration, the energy value at a given range was calculated as a sum of products of Planck constant, number of activated photons, and frequency, which corresponds to the wavelength. The mathematical expression of radiation energies $E_{\mathrm{UV}}, E_{\mathrm{VIS}}$, and $E_{\mathrm{NIR}}$ is given by

$$
\begin{aligned}
& E_{\mathrm{UV}}=\sum_{i=f_{1}}^{f_{2}} n_{\mathrm{UV}} h f_{i} \\
& E_{\mathrm{VIS}}=\sum_{i=f_{1}}^{f_{2}} n_{\mathrm{VIS}} h f_{i} \\
& E_{\mathrm{NIR}}=\sum_{i=f_{1}}^{f_{2}} n_{\mathrm{NIR}} h f_{i},
\end{aligned}
$$

where $E_{\mathrm{UV}}, E_{\mathrm{VIS}}$, and $E_{\mathrm{NIR}}$ are radiation energy in the UV, VIS, and NIR range, respectively [J], $f_{1}$ is frequency at which the considered range begins $[\mathrm{Hz}], f_{2}$ is frequency at which the considered range ends $[\mathrm{Hz}]$, and $n_{\mathrm{UV}}=130, n_{\mathrm{VIS}}=95$, and $n_{\mathrm{NIR}}=60$ are number of photons in the UV, VIS, and NIR range, respectively.

Afterwards, the total radiation energy $E_{C}$ for the whole analyzed electromagnetic radiation spectrum was determined according to [21]

$$
E_{\mathrm{C}}=E_{\mathrm{UV}}+E_{\mathrm{VIS}}+E_{\mathrm{NIR}}
$$

Based on the total radiation energy $E_{C}$ the approximate percentage contribution of individual energies was estimated as $\% E_{C}$. Table 3 presents the calculated energy values for the considered ranges.

For better analysis, the radiation energy values were depicted graphically in Figure 9, presenting also the result 

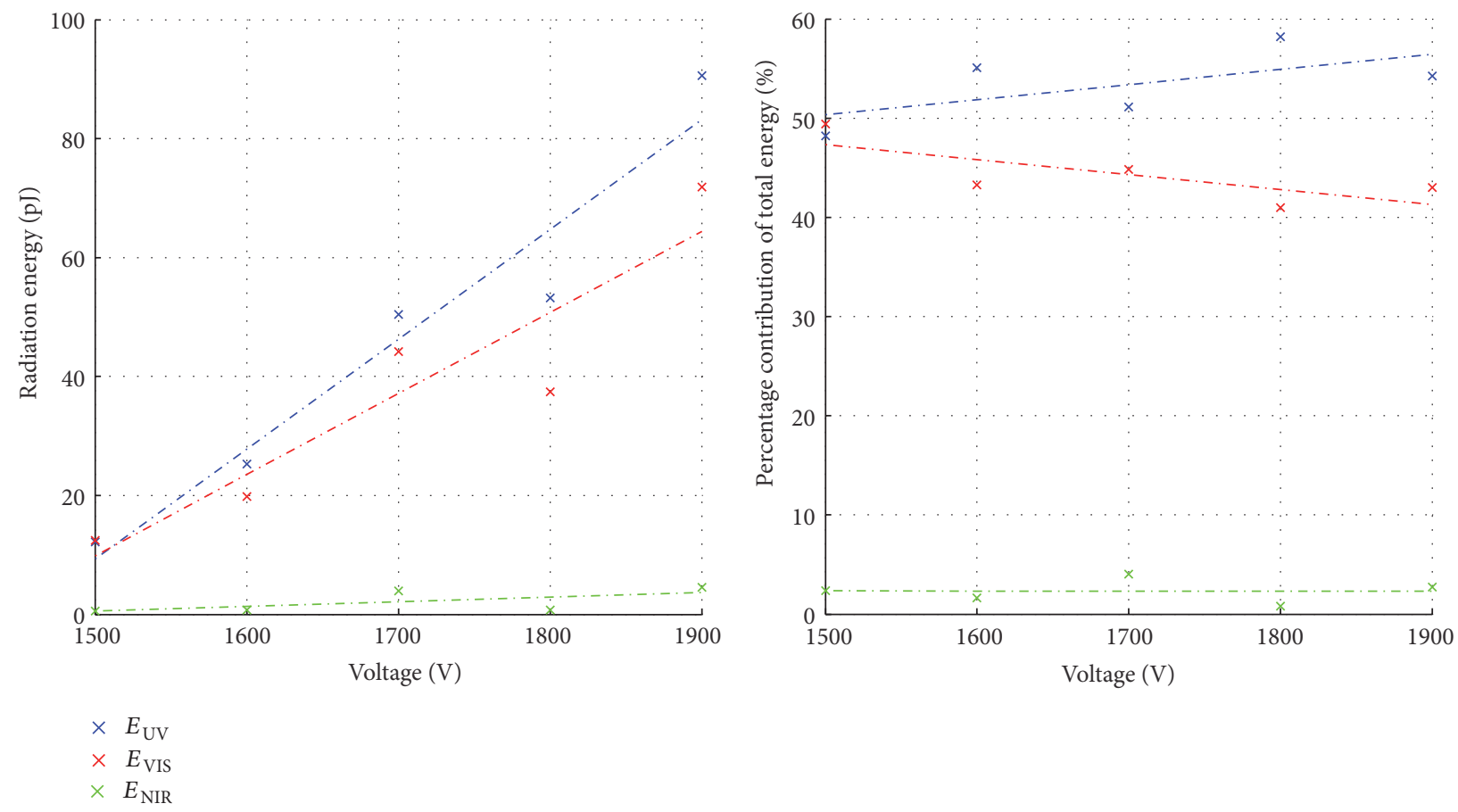

FIGURE 9: Dependency of voltage on the calculated electromagnetic radiation energy values.

of linear regression. One may recognize a significant dependency of voltage value on the radiation energy contained in the VIS and UV ranges and that the energy contained in the NIR range is the lowest, although it also increases according to the rising voltage value.

The percentage contribution of total energy $\% E_{C}$ for the UV, VIS, and NIR ranges remains stable with a slight decrease of $E_{\mathrm{VIS}}$ for the benefit of the increase of $E_{\mathrm{UV}}$ at higher voltage values. A considerable voltage independent low energy concentration occurs within the NIR range. The radiation of UV has the highest energy regardless of supply voltage value.

\section{Conclusions and Summary}

During the research, the application possibility of optical spectrophotometry for detection and analysis of free burning electric arc phenomenon, generated by various voltage values in the air, was confirmed. For several measurements, performed under the same laboratory conditions, the radiation spectra ranging from $200 \mathrm{~nm}$ to $1100 \mathrm{~nm}$ were obtained. Based on the performed analyses of the registered optical radiation spectra, the following can be stated:

(i) The dominant frequency components of significantly higher intensity were recognized in the registered optical signals. The number of significant wavelengths is wider as the supply voltage increases. The amplitudes rise in accordance with the voltage values.

(ii) The obtained coefficients, peak and shape factors, may be applied as potential benchmarks for the identification of electric arc purposes regardless of voltage value, and it may be crucial for diagnosis of electrical apparatus.

(iii) The most radiation energy is detected in the UV and VIS ranges, while it is slightly lower for the VIS range, regardless of the voltage value. On average only $2.32 \%$ of the total energy is detected within the NIR range. In the whole analyzed optical spectrum, the estimated energy values increase according to the rising supply voltage value but the most significant increase was recognized for the UV range

It is to mention that the presented experimental results constitute the very first stage of research aimed at developing a database of various descriptors, comprising unique criteria for identification and characterization of electrical discharges including free burning arcs in the air. Such a database, which includes a series of the so-called "finger prints", each corresponding to various defects of the electrical apparatus, may be used in expert systems for diagnostic purposes. The obtained results have potential to be applied to increase the accuracy and efficiency of diagnostic systems used for evaluation of the technical condition of electrical circuits such as an electric arc safety device.

The presented method of spectral analysis of optical signals emitted by an electric arc may, at the subsequent stage of the research, be applied for various types of materials and designs of electrodes, commonly used in electrical engineering. It is also planned to determine the impact of additional factors, such as atmospheric conditions or insulator type on the obtained results. Such research study will make it possible to select the most effective method for measurement and 
analysis of electrical discharges for the purpose of diagnostics of electric power systems.

\section{Competing Interests}

The authors declare that there is no conflict of interests regarding the publication of this paper.

\section{References}

[1] V. Chernyak, V. Naumov, and V. Yukhimenko, "Spectroscopic of atmospheric pressure air jet plasma in transverses arc discharge," in Proceedings of the 12th International Congress on Plasma Physics, Nice, France, October 2004.

[2] W. Tarczyñski and T. Daszkiewicz, "Dynamics of discharge channel displacement in AC electric arcs," Archives of Electrical Engineering, vol. 58, no. 3-4, pp. 127-142, 2009.

[3] D. Hoseini, R. Shourvarzi, and M. M. Rostami, "Effect of electric arc resistance on the transition state due to the breakers switching in substations with air insulation," in Proceedings of the 7th International Power Electronics and Motion Control Conference (IPEMC '12), vol. 4, pp. 2647-2651, Harbin, China, 2012.

[4] P. Slade, Electrical Contacts: Principles and Applications, Taylor \& Francis, Oxford, UK, 2nd edition, 2014.

[5] M. Cernan and J. Tlusty, "Model of Electric Arc Furnace For Designing Of Power Quality Improvement Equipment," in Proceedings of the International Scientific Conference on Electric Power Engineering, pp. 187-192, Brno, Czech Republic, 2014.

[6] B. Barbu, A. Iturregi, F. Berger, and E. Torres, "Numerical analysis of the electric arc simulation using ansys CFX," in Proceedings of the 26th International Conference on Electrical Contacts (ICEC '12), pp. 311-316, IEEE, Beijing, China, May 2012.

[7] S. Gu, J. He, B. Zhang, G. Xu, and S. Han, "Movement simulation of long electric arc along the surface of insulator string in free air," IEEE Transactions on Magnetics, vol. 42, no. 4, pp. 13591362, 2006

[8] L. Hongru, Z. Haoy, L. Fangyan, and Q. Bo, "A hybrid simulation model of AC electric arc furnace," in Proceedings of the 24th Chinese Control and Decision Conference (CCDC '12), pp. 188193, Taiyuan, China, May 2012.

[9] V. Correcher, J. García-Guinea, C. Martin-Fernandez, and N. Can, "Thermal effect on the cathodo- and thermoluminescence emission of natural topaz $\left(\mathrm{Al}_{2} \mathrm{SiO}_{4}(\mathrm{~F}, \mathrm{OH})_{2}\right)$," Spectroscopy Letters, vol. 44, no. 7-8, pp. 486-489, 2011.

[10] A. J. Wu, H. Zhang, X. D. Li, S. Y. Lu, C. M. Du, and J. H. Yan, "Determination of spectroscopic temperatures and electron density in rotating gliding arc discharge," IEEE Transactions on Plasma Science, vol. 43, no. 3, pp. 836-845, 2015.

[11] Z. X. Wang, J. W. Spencer, J. D. Yan, G. R. Jones, and J. E. Humphries, "Preliminary spectroscopic investigation of HVCB contacts erosion," Plasma Physics and Technology, vol. 2, no. 2, pp. 203-206, 2015.

[12] M. Muhr and R. Schwarz, "Experience with optical partial discharge detection," Materials Science-Poland, vol. 27, no. 4, pp. 1139-1146, 2009.

[13] R. Badent, K. Kist, A. J. Schwab, and M. Wurster, "Light emission measurements of predischarges in insulating oil," in Proceedings of the 67th IEEE Annual Conference on Electrical Insulation and Dielectric Phenomena, vol. 2, pp. 452-455, Atlanta, Ga, USA, October 1998.
[14] P. Frącz, "Measurement of optical signals emitted by surface discharges on bushing and post insulator," IEEE Transactions on Dielectrics and Electrical Insulation, vol. 20, no. 5, pp. 1909-1914, 2013.

[15] P. Fracz, T. Boczar, and S. Borucki, "Analysis of optical radiation generated by electrical discharges on support insulator," Acta Physica Polonica A, vol. 124, pp. 413-416, 2013.

[16] S. A. Popov, A. V. Batrakov, R. Methling, D. Uhrlandt, and K. Weltmann, "Spectroscopic study of the anode flare formation during the initial stage of vacuum arc discharge," in Proceedings of the IEEE International Conference on Plasma Sciences (ICOPS '15), p. 1, IEEE, Antalya, Turkey, May 2015.

[17] J. Lane, C. Jiang, J. Sanders, A. Kuthi, and M. A. Gundersen, "Spatially resolved optical emission spectroscopy of a nanosecond pulsed microplasma jet," in Proceedings of the 41st IEEE International Conference on Plasma Science (ICOPS '14) and the 20th IEEE International Conference on High-Power Particle Beams (BEAMS '14), Washington, DC, USA, May 2014.

[18] M. Simek, "Electrical and optical characteristics of surface DBD in pure N2 and N2-NO mixtures," in Proceedings of the IEEE International Conference on Plasma Science (ICOPS '09), pp. 15, San Diego, Calif, USA, June 2009.

[19] Ocean Optics, "HR4000 and HR4000CG-UV-NIR Series Spectrometers Installation and Operation Manual," http://oceanoptics.com///wp-content/uploads/hr4000.pdf.

[20] M. Alonso and E. J. Finn, Fundamental University Physic, Volume III: Quantum and Statistical Physics, Addison-Wesley, 1968.

[21] X. Ma, C. Zhou, and I. J. Kemp, "Interpretation of wavelet analysis and its application in partial discharge detection," IEEE Transactions on Dielectrics and Electrical Insulation, vol. 9, no. 3, pp. 446-457, 2002. 

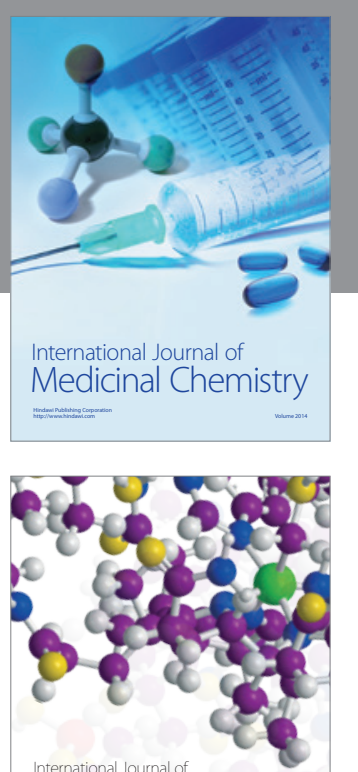

Carbohydrate Chemistry

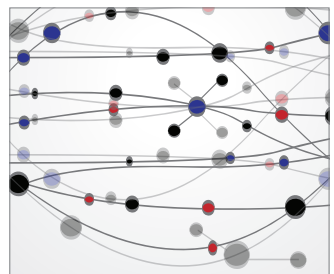

The Scientific World Journal
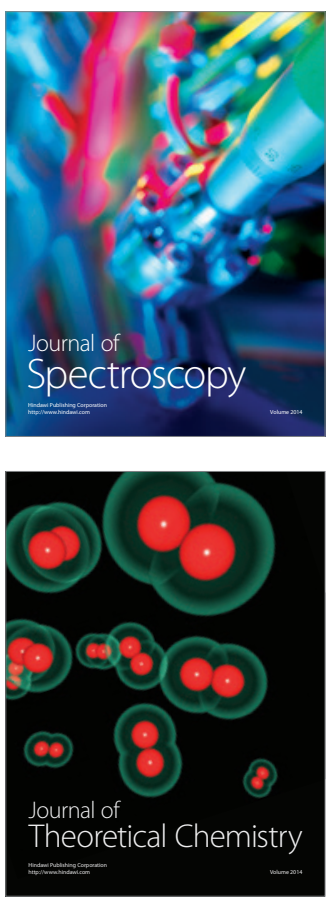
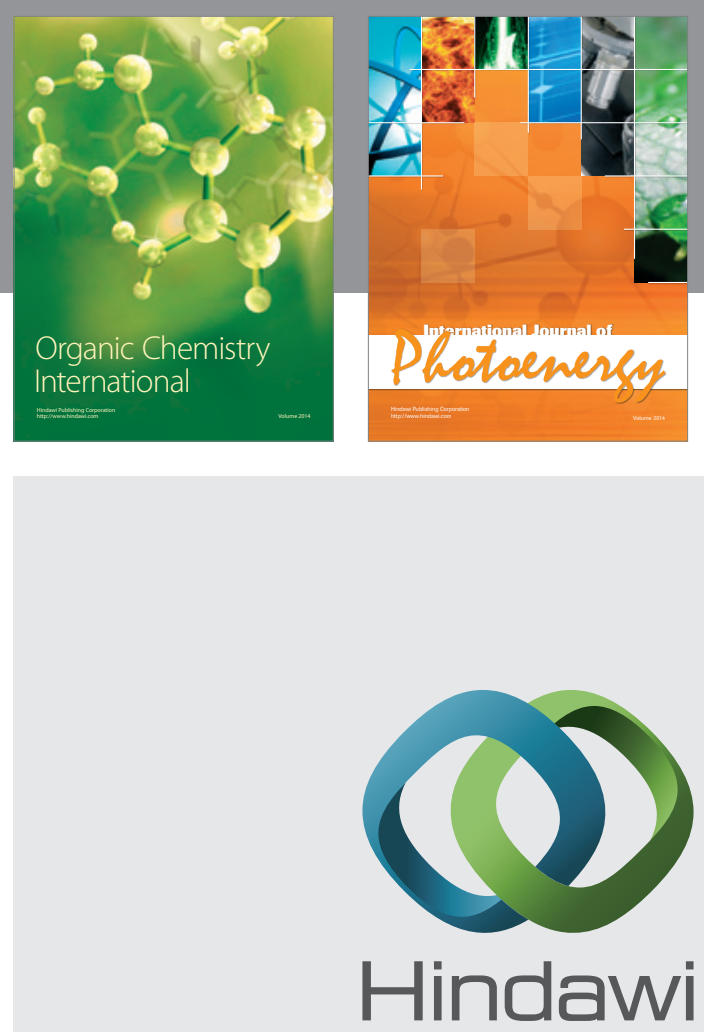

Submit your manuscripts at

http://www.hindawi.com

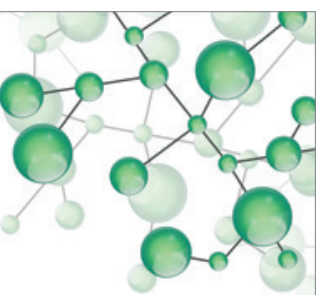

International Journal of

Inorganic Chemistry

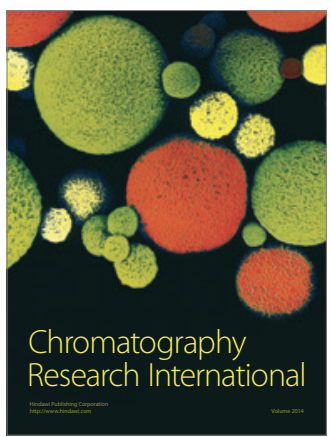

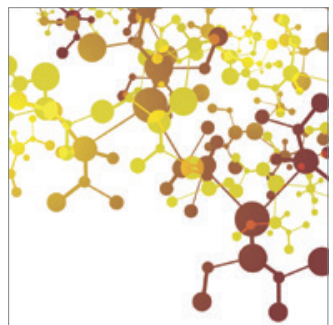

Applied Chemistry
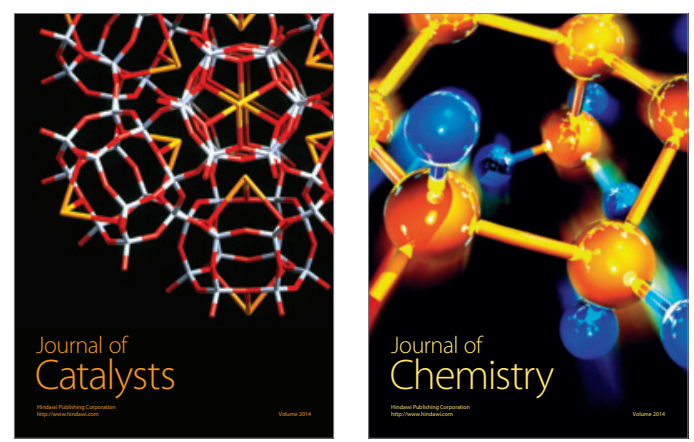
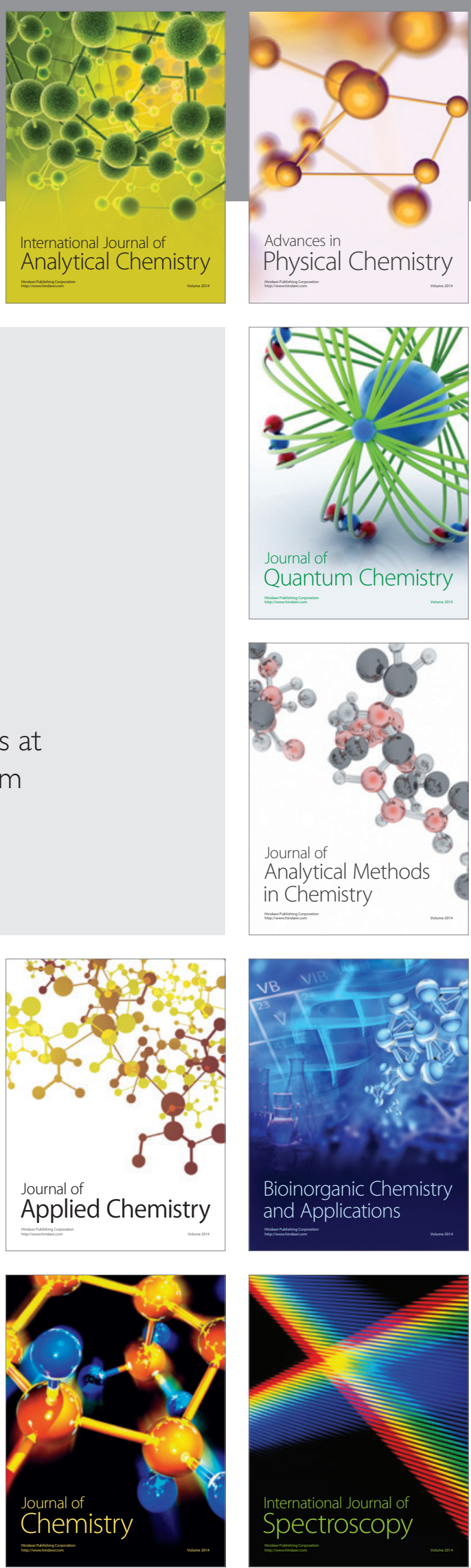